SLAC-PUB-12249

December 2006

(ACCPHY/MATSCI)

\title{
Improved Superlattices for Spin-Polarized Electron Sources *
}

\author{
Yu. A. Mamaev, L. G. Gerchikov, Yu. P. Yashin, V. Kuz'michev, D. Vasiliev \\ State Polytechnic University, Politekhnicheskaya str., 29, 195251, St.-Petersburg, Russia
}

T. Maruyama, J. E. Clendenin

Stanford Linear Accelerator Center, 2575 Sand Hill Road, Menlo Park, CA 94025, USA

V.M.Ustinov and A.E.Zhukov

Ioffe Physico-Technical Institute, RAS, 194021, St. Petersburg, Russia

\begin{abstract}
Photoemission of polarized electrons from heterostructures based on InAlGaAs/GaAs superlattices with minimum conduction-band offsets is investigated. The comparison of the excitation energy dependence of the photoemission polarization degree with the calculated spectra makes it possible to determine the polarization losses at different stages of the photoemission. A maximum polarization of $P=91 \%$ and a quantum efficiency of $Q E=0.5 \%$ are close to the best results obtained for photocathodes that are based on strained semiconductor superlattices.
\end{abstract}

Contributed to

The $17^{\text {th }}$ International Spin Physics Symposium (SPIN 2006))

October 2-7, 2006, Kyoto, Japan

\footnotetext{
* Work supported in part by U.S. Department of Energy under contract DE-AC02-76SF00515.
} 


\title{
Improved Superlattices for Spin-Polarized Electron Sources
}

\author{
Yu. A. Mamaev, L. G. Gerchikov, Yu. P. Yashin, V. Kuz'michev, \\ D. Vasiliev
}

State Polytechnic University, Politekhnicheskaya str., 29, 195251, St.-Petersburg, Russia

T. Maruyama, J. E. Clendenin

Stanford Linear Accelerator Center, 2575 Sand Hill Road, Menlo Park, CA 94025, USA

V.M.Ustinov and A.E.Zhukov

Ioffe Physico-Technical Institute, RAS, 194021, St. Petersburg, Russia

\begin{abstract}
Photoemission of polarized electrons from heterostructures based on InAlGaAs/GaAs superlattices with minimum conduction-band offsets is investigated. The comparison of the excitation energy dependence of the photoemission polarization degree with the calculated spectra makes it possible to determine the polarization losses at different stages of the photoemission. A maximum polarization of $P=91 \%$ and a quantum efficiency of $Q E=0.5 \%$ are close to the best results obtained for photocathodes that are based on strained semiconductor superlattices.
\end{abstract}

Keywords: superlattice, minimum conduction-band offsets, photoemission.

PACS: 72.25.Fe,73.21.Cd,79.60.-i

\section{MOTIVATION}

In a real semiconductor heterostructure the maximum polarization of photoelectrons is limited by broadening of the valence band energy spectrum and smearing of the valence-band edge. This causes the mixing of light and heavy holes even at the absorption edge so that the observed polarization is $P<100 \%$. The mixing of states of light and heavy holes can be reduced by increasing the energy of splitting between the $h h$ and $l h$ subbands.

In strained superlattices (SLs), the splitting $E_{h h-l h}$ of the subbands of light and heavy holes appears because of the heterolayer deformations and due to quantum confinement; the strain-induced splitting and the splitting caused by quantum confinement are not additive. The resulting splitting depends in a complicated way on the composition and thickness of the SL layers. The composition of the layers 
determines their strain and the band offsets at the heterointerfaces, while the layer thickness affects the quantum confinement energy and SL barrier transparence. Thus, choosing the optimal structure of a SL to provide a large $E_{h h-l h}$ splitting in the absence of stress relaxation and in combination with good transport properties is a difficult problem. The solution requires a reliable method to calculate the energy spectrum of the SLs on the basis of ternary and quaternary III-V semiconductor compounds. In addition, the maximum photoelectron polarization depends crucially on the structural quality of the SLs, which imposes heightened requirements on the technology of their growth.

The study of the properties of short-period strained SLs and the development of the technology of their growth achieved in the last decade have made it possible to create photocathodes with record breaking values of photoemission polarization of $P \approx 90 \%$. Photocathodes based on GaAs/GaAsP [1] and InGaAs/GaAlAs [2] SL structures with strained quantum wells, GaAs/AlInGaAs-based SLs with strained barriers, and socalled compensated SLs based on AlInGaAs/GaAsP structures with opposite strains in the wells and barriers [3] have been developed. These studies have also revealed fundamental difficulties for achieving further progress. The valence band splitting $E_{h h-}$ $I_{h}$ in an SL cannot exceed the valence-band offsets. Therefore, to create a significant splitting $E_{h h-l h} \approx 100 \mathrm{meV}$, an SL with large band offsets at the heterointerfaces must be used. In this case, high barriers (of hundreds of $\mathrm{meV}$ ) usually appear in the conduction band that complicate the transport of photoelectrons to the surface of the photocathode. Low electron mobility along the SL axis reduces the QE. In turn, this leads to a partial loss of spin orientation by electrons during their transport to the surface due to the D'yakonov-Perel' mechanism [4] for spin relaxation. One can minimize the barriers in the conduction band in a GaAs/AlInGaAs-based SL by selecting the composition of the quaternary AlInGaAs compound. At the same time, the structure of the valence band allows one to obtain the splitting $E_{h h-l h}$ as large as 70 $\mathrm{meV}$. In this way, for SL photocathodes with minimum conduction-band offsets, a record-breaking electron emission polarization of $P=91 \%$ has been achieved [5].

\section{RESULTS AND DISCUSSION}

We have studied a series of photoemitters based on SLs with minimum conductionband offsets. We analyzed the spectra of photoemission and polarization for cathodes with different compositions and SL layer thicknesses. By varying the SL parameters we could change the position of the polarization maximum in the range of photon energies from 1.44 to $1.52 \mathrm{eV}$. In all samples, high polarization of the electronic emission of $84 \%<P<91 \%$ was observed at a $Q E$ of $0.05 \%<Q E<0.5 \%$. 
TABLE 1. Photocathode structure.

\begin{tabular}{|c|c|c|c|}
\hline \multicolumn{2}{|c|}{ Composition } & Thickness & Doping \\
\hline \multicolumn{4}{|c|}{ As cap } \\
\hline GaAs & Quantum well & $6 \mathrm{~nm}$ & $7 * 10^{18} \mathrm{~cm}^{-3}(\mathrm{Be})$ \\
\hline $\mathrm{In}_{\mathrm{x}} \mathrm{Al}_{\mathrm{y}} \mathrm{Ga}_{1-\mathrm{x}-\mathrm{y}} \mathrm{As}$ & \multirow{2}{*}{ Superlattice } & $3-4 \mathrm{~nm}$ & \multirow[t]{2}{*}{$4 * 10^{17} \mathrm{~cm}^{-3}(\mathrm{Be})$} \\
\hline GaAs & & $1.1-1.5 \mathrm{~nm}$ & \\
\hline $\mathrm{Al}_{0.35} \mathrm{Ga}_{0.65} \mathrm{As}$ & Buffer & $0.3-1.4 \mu \mathrm{m}$ & $6^{*} 10^{17} \mathrm{~cm}^{-3}(\mathrm{Be})$ \\
\hline \multicolumn{4}{|c|}{ GaAs (100) - wafer, p-doped } \\
\hline
\end{tabular}

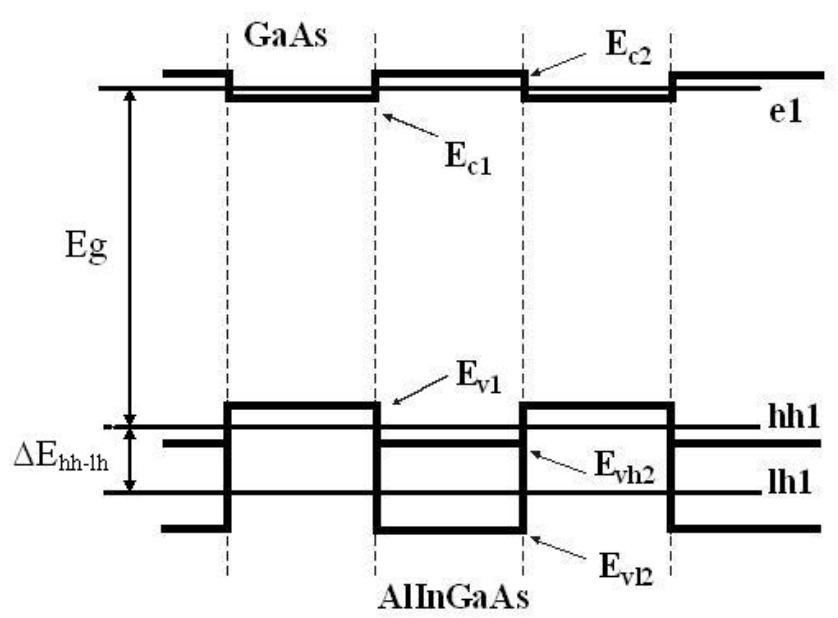

FIGURE 1. Profiles of the conduction-band edge $E_{c}$ and valence band edge $E_{v}$ in the SL. In the AlInGaAs layer, the valence band is split into the subbands of heavy holes $v_{h 2}$ and light holes $v_{l 2}$. Thin horizontal lines indicate the positions of the lower electron miniband $e l$ and the minibands of heavy holes hh1 and light holes lh1.

A typical photocathode structure is shown in Table 1. The composition and thickness of the SL layers for the samples under study are specified in the table. Their values were chosen to maximize the $E_{h h-l h}$ splittings and minimize the conduction 
band offset in the absence of strain relaxation for a given In concentration. A typical profile of the conduction band edge $E_{c}$ and the light hole $E_{v l}$ and heavy hole $E_{v h}$ band edges is shown in Fig. 1. The lattice constant in the SL layer plane is determined by the GaAs substrate and the buffer layer. Therefore, the GaAs layer in the SL is not strained, whereas the InAlGaAs layer is compressed in the layer plane and stretched along the SL axis.

The quantum confinement energies and the splitting energy $E_{h h-l h}$ depend on the barrier thickness. We have performed the calculations of the energy spectrum of the SL using the envelope method and the Kane four-band model including the conduction band, valence bands, and spin-orbit split-off band [6]. Figure 2 shows the dependence of the hole miniband edges $E_{\mathrm{hh} 1}$ and $E_{\mathrm{lh} 1}$ on the width $a$ of a GaAs well and on the thickness $b$ of an $\operatorname{In}_{x} \mathrm{Al}_{\mathrm{y}} \mathrm{Ga}_{1-\mathrm{x}-\mathrm{y}} \mathrm{As}$ barrier for the concentrations $x=0.2$ and $y=0.23$. We see that the quantity $E_{h h-l h}=E_{h h 1}-E_{l h 1}$ increases with increasing $b$ and decreasing $a$ until the limiting value of the strain splitting is reached. The choice of the values of $a$ and $b$ is the result of a compromise between the desire to attain the maximum value of $E_{h h-l h}$ and the requirement to grow the SL without dislocations and structural defects.

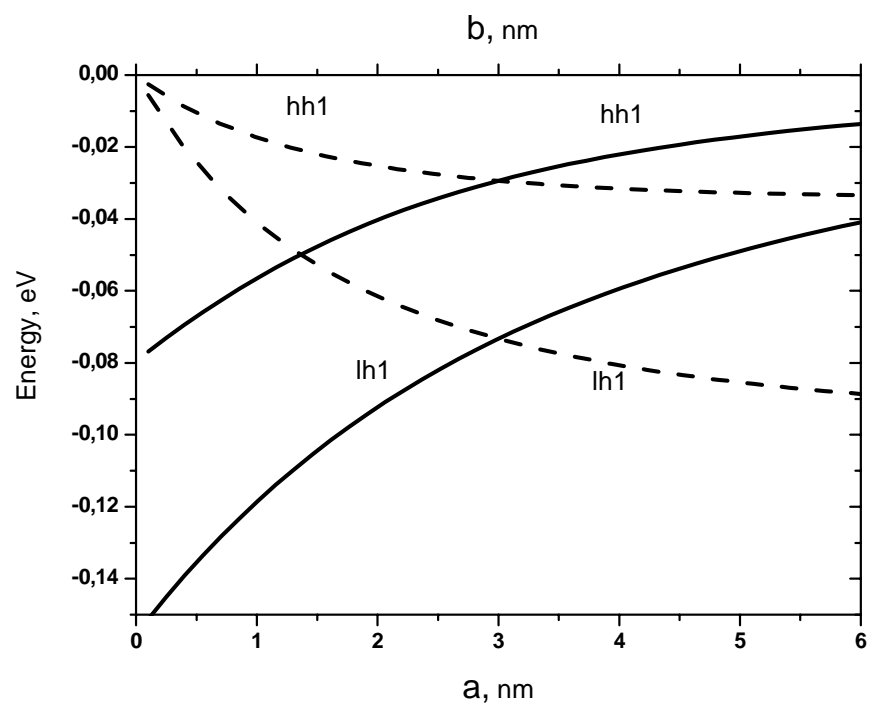

FIGURE 2. Dependences of the position of the edges of the minibands of light holes $\left(\mathrm{E}_{\mathrm{lh} 1}\right)$ and heavy holes $\left(\mathrm{E}_{\mathrm{hh} 1}\right)$ on the width $a$ of GaAs wells (solid lines) and on the thickness $b$ of $\operatorname{In}_{0.2} \mathrm{Al}_{0.23} \mathrm{Ga}_{0.57} \mathrm{As}$ barriers (dashed lines)

All samples were grown by molecular beam epitaxy in a RIBER 32P installation. The growth rates were preliminarily calibrated by analyzing the photoluminescence spectrum of the test sample grown before the series of structures under study.

In Fig. 3, the experimental spectra of $Q E$ and $P$ for a sample based on an $\mathrm{In}_{0.25} \mathrm{Al}_{0.27} \mathrm{Ga}_{0.48} \mathrm{As}(4 \mathrm{~nm}) / \mathrm{GaAs}(1.1 \mathrm{~nm})$ SL are compared with the results of the calculations. Vertical lines mark the positions of the absorption edges for hh1-e1 and lh1-e1 transitions from the minibands of heavy and light holes respectively to the conduction band. Using this construction, it is easy to understand the behavior of the 
$P(\hbar \omega)$ and $Q E(\hbar \omega)$ spectra. Maximum polarization as well as the threshold feature in the $Q E(\hbar \omega)$ spectrum appear at the absorption edge of hh1-e1 transitions where the effect of the lh1-e1 transitions is minimal. With increasing photon energy, the polarization of photoelectrons decreases due to optical transitions from the of light hole states $l h 1$. The region of the sharpest decrease in polarization coincides with the threshold for lh1-e1 transitions.

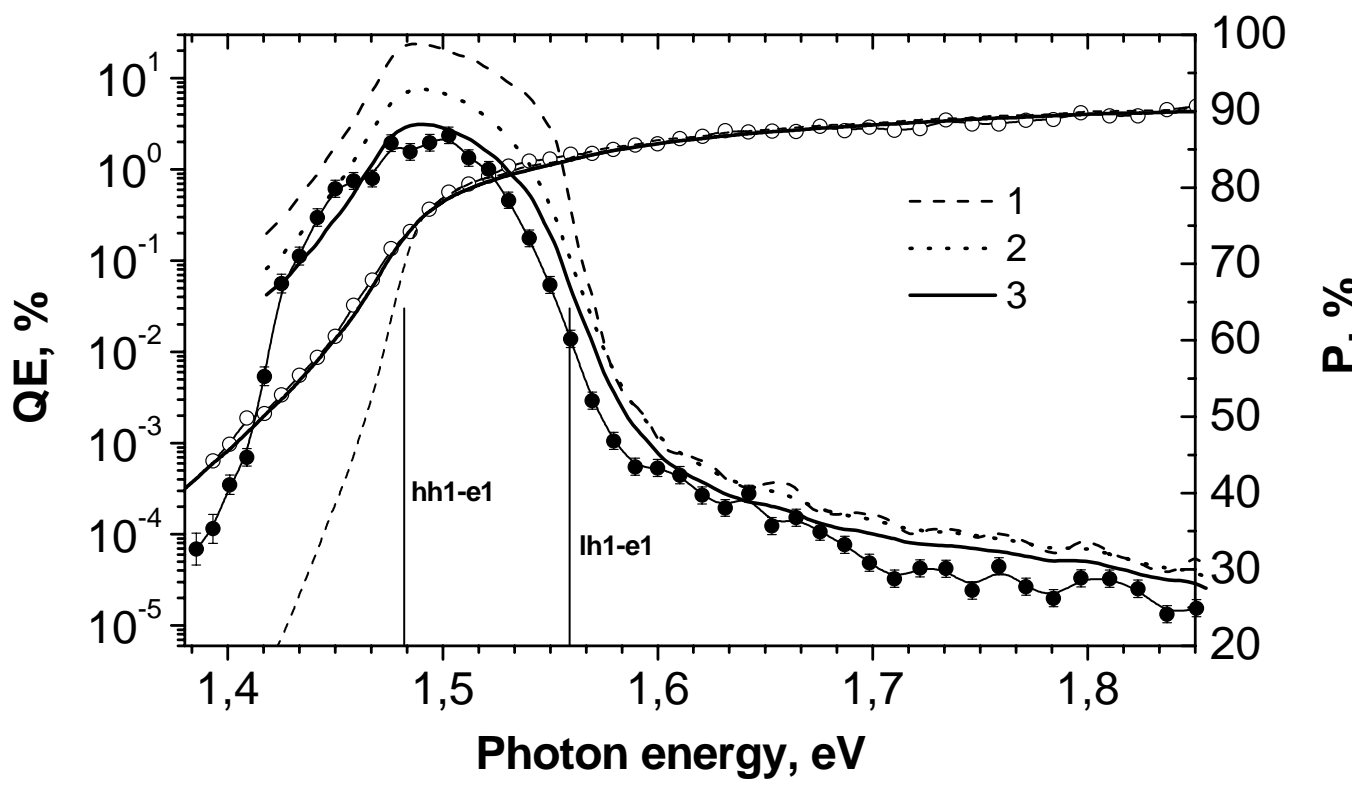

FIGURE 3. Spectra of the polarization and $Q E$ for sample with $\operatorname{In}_{0.25} \mathrm{Al}_{0.27} \mathrm{Ga}_{0.48} \mathrm{As}(4 \mathrm{~nm}) / \mathrm{GaAs}(1.1 \mathrm{~nm})$ SL. Experimental results: closed circles - polarization, open circles - quantum efficiency. A thin line connects the data points. Results of the calculation: (1) $\gamma=\delta=10 \mathrm{meV}$, (2) $\gamma=\delta=30 \mathrm{meV}$, (3) $\gamma=\delta=$ $30 \mathrm{meV}$ with polarization losses $B_{\mathrm{s}}=5 \%$.

To analyze these results we calculated both the initial polarization $P_{0}$ and the generation rate of photoelectrons produced in the SL by circularly polarized light. In our calculations we take into account the broadening of the hole spectrum and the tails of the density of hole states in the band gap [6,7]. In Fig. 3 the dashed and dotted lines show the dependence of initial electron polarization $P_{0}(\hbar \omega)$ calculated for two different values of the hole spectrum broadening $\gamma=10$ and $30 \mathrm{meV}$. We see that the increase in $\gamma$ reduces the value of the initial electron polarization $P_{0}$ at the maximum by $6 \%$. Another parameter affecting the behavior of the absorption spectrum near the edge $\hbar \omega=E_{g}$ is the extent $\delta$ of the tails of the hole states in the band gap, i.e., the broadening of the absorption edge. This parameter has a more profound effect on the dependence $Q E(\hbar \omega)$ below the absorption threshold (see Fig. 3 dashed, $\delta=10 \mathrm{meV}$, and solid, $\delta=30 \mathrm{meV}$, curves $Q E(\hbar \omega))$. A comparison of the calculated photoabsorption rate with the experimentally observed $Q E$ allows us to determine the values of the 
following two parameters of the sample: (1) the slope $Q E(\hbar \omega)$ below the photoabsorption threshold gives us the value of $\delta=30 \mathrm{meV}$; and (2) the absolute value of the $Q E$ above the photoabsorption threshold gives us the probability of electron emission into vacuum of $\approx 9 \%$. We estimated the values of $\gamma$ and the polarization losses $B_{s}$ during electron transport to the surface and emission into the vacuum by comparing the theoretical spectrum of initial polarization $P_{0}(\hbar \omega)$ with the experimental $P(\hbar \omega)$ curve. The choice of $\gamma=30 \mathrm{meV}$ and $B_{s}=5 \%$ brings the calculated polarization curve (solid line in Fig. 3) close to the experimental result of $P_{\max }=87 \%$. The rather large values of $\gamma$ and $\delta$ are caused by the fluctuations of the heterolayer composition. As a result the maximal initial polarization $P_{0}$ in this sample is only $91 \%$. It should be noted that the initial photoelectron polarization losses are almost twice as large as the $5 \%$ polarization losses in the course of emission.

\section{ACKNOWLEDGMENTS}

This work was supported by RFBR under grant 04-02-16038, NATO under grant PST.CLG.979966, the U.S. Department of Energy under contract DE-AC0276SF00515, Swiss National Science Foundation under grant SNSF IB7420-111116 and by Russian Ministry of Education and Science under grant N.P.2.1.1.2215.

\section{REFERENCES}

1. Yu.Mamaev, A. Subashiev, Yu. Yashin, E. Reichert, P. Drescher, N. Faleev, P. Kop'ev, V. Ustinov and A. Zhukov, Phys. Low-Dimens. Struct. 10/11, 61 (1995).

2. T. Maruyama, D.-A. Luh, A. Brachmann, J. E. Clendenin, E. L. Garwin, S. Harvey, J. Jiang, R. E. Kirby, and C. Y. Prescott, Appl. Phys. Lett. 85, 2640 (2004).

3. A. V. Subashiev, L. G. Gerchikov, Yu. A. Mamaev, Yu. P. Yashin, J. S. Roberts, D.-A. Luh, T. Maruyama, and J. E. Clendenin, Appl. Phys. Lett. 86, 171911 (2005).

4. Optical Orientation, edited by F. Meier and B. P. Zakharchenya, North-Holland, Amsterdam, 1984.

5. Yu.A.Mamaev, A.V.Subashiev, Yu.P.Yashin, T. Maruyama, D.-A. Luh, J.E. Clendenin, V.M. Ustinov, A.E. Zhukov, SPIN 2004 Proceedings, World Scientific, Singapore, 2005, p. 913.

6. A.V. Subashiev, L.G. Gerchikov, and A.I. Ipatov, J. Appl. Phys. 96, 1511 (2004).

7. A.V. Subashiev and L.G. Gerchikov, SPIN 2004 Proc. (World Scientific, 2005) p. 908. 